\title{
A robust multi color lane marking detection approach for Indian scenario
}

\author{
L N P Boggavarapu, R S Vaddi, \\ K R Anne \\ Department of Information \\ Technology, \\ V R Siddhartha Engineering College, \\ Vijayawada.
}

\author{
H D Vankayalapati \\ Department of Computer Science \\ Engineering \\ V R Siddhartha Engineering \\ College, Vijayawada.
}

\author{
J K Munagala \\ Department of Electronics \& \\ Computers \\ PVP Siddhartha Institute of \\ Technology, Vijayawada
}

\begin{abstract}
Lane detection is an essential component of Advanced Driver Assistance System. The cognition on the roads is increasing day by day due to increase in the four wheelers on the road. The cognition coupled with ignorance towards road rules is contributing to road accidents. The lane marking violence is one of the major causes for accidents on highways in India. In this work we have designed and implemented an automatic lane marking violence detection algorithm in real time. The HSV color-segmentation based approach is verified for both white lanes and yellow lanes in Indian context. Various comparative experimental results show that the proposed approach is very effective in the lane detection and can be implemented in realtime.
\end{abstract}

Keywords- Color segmentation; HSV; Edge orientation; connected components.

\section{INTRODUCTION}

Traffic accidents have become one of the most serious problems in today's world. Roads are the choicest and most opted modes of transport in providing the finest connections among all other modes [1]. Due to increase in vehicles from $3,00,000$ in 1951 to about 7,30,00,000 in 2004 [2] as shown in Fig 1, traffic accidents especially road accidents have become predominant. According to official statistics 105,725 people were killed in road traffic crashes in India in 2006 (NCRB, 2007) [3]. During recent years, traffic fatalities increased by about 5 percent per year from 1980 to 2000 [2] and since then have increased by about 8 percent per year for the four years for which statistics are available as shown in Fig 2.

The major factors that contribute to road accidents are due to negligence of the driver. Reducing the accidents on road is possible by improving the road safety. Increasing the safety and saving lives of human beings is one of the basic features in Advanced Driver Assistance System (ADAS), a component in Intelligent Transportation System (ITS). A real time computer vision based system plays an important role in providing a useful and effective information like lane marking [20], departure and front and side images etc. The present paper deals with the detection of lanes on roads especially Indian typical roads.
Many researchers have shown lane detectors based on a wide variety of techniques. Techniques used varied from using monocular to stereo vision using low level morphological operations to using probabilistic grouping and B-snakes [22]. All the techniques are classified into two main categories namely feature based techniques and model based techniques. The feature based technique combines low level features like color; shape etc. in order to detect the lane and the modelbased scheme is more robust in lane detection when different lane types with occlusions or shadows are handled. Road and lane markings can vary greatly, making the generation of a single feature-extraction technique is difficult. So, we combined the features of both color based and edge based techniques [23], [24].

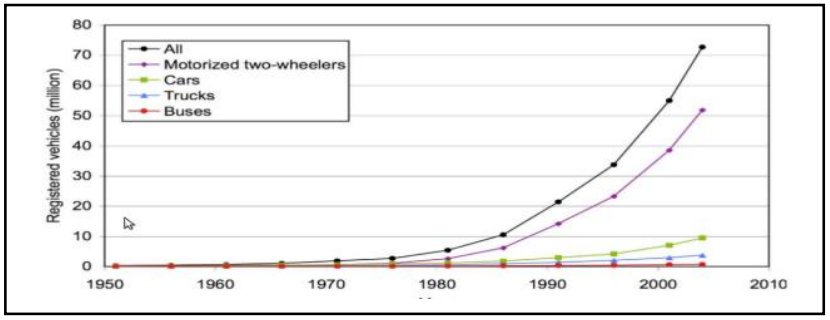

Figure 1. Registered vehicles, 1951 through 2004

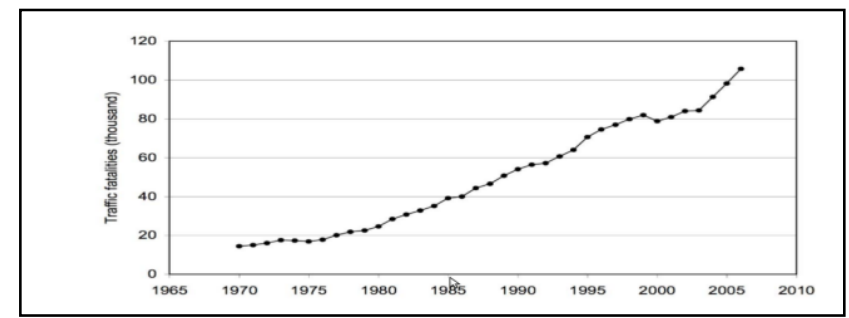

Figure 2. Traffic fatalities, 1970 through 2006

\section{STATE OF ART}

During the Recent years, many techniques for processing and analyzing images for lane detection have been proposed which involves, Template Matching and Ellipse Modeling Approach to Detecting Lane Markers [4]. Lakshmanan and 
Kluge [5] applied deformable template model of lane structure to locate lane boundaries without thresholding the intensity gradient information. Yim and Oh [6] developed a threefeature-based automatic lane-detection algorithm using the starting position, direction, and gray-level value of a lane boundary as features to recognize the lane. ZuWhan Kim [7] developed new method for lane detection which involves lane boundary hypotheses generation and probabilistic lane grouping. M. Bertozzi and A. Broggi [8] were proposed a method for lane-detection which is based on morphological filter. It is observed from literature study that only very few attempts have been made to work on color images [9]. Yu-Chi Leng and Chieh-Li Chen [10] proposed a method for lanedetection which is based on urban traffic scenes [20]. Yue Wang, Eam Khwang Teoh and Dinggang Shen developed Lane detection and tracking using B-Snake [22]. H. Zhang, et al proposed a Novel Lane Detection Algorithm Based on Support Vector Machine.[21]. In the present study, two lane features, lane width and lane boundary continuity, are proposed to obtain reliable and quality lane detected results.

\section{LANE MARKING DETECTION USING HSV-EDGE BASED} APPROACH

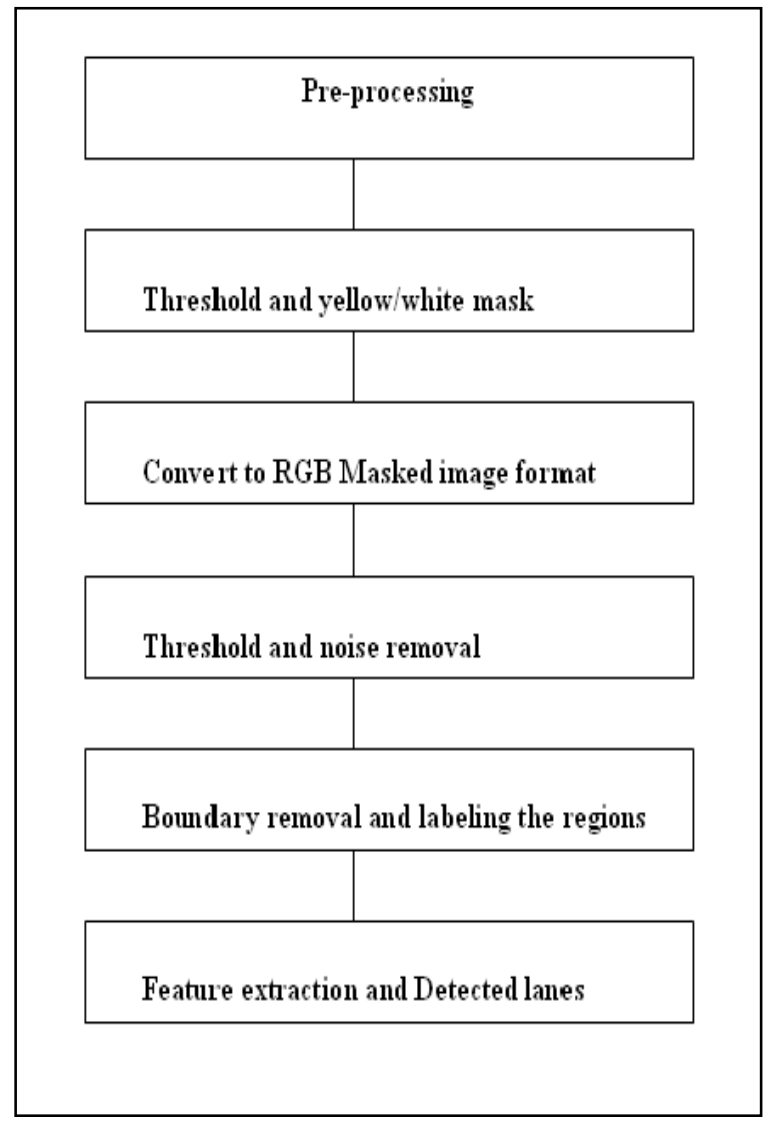

Figure 3. Proposed approach

Many lane detection approaches [17], [18], [19] use color model in order to segment the lane line from background images. However, the color feature [2] is not sufficient to decide an exact lane line in images depicting the variety of road markings and conditions. If there are many lanes or obstacle which is similar to lane color, it will be difficult to decide an exact lane. Similarly, some lane detection method uses only edge information. The proposed method involves the combination of both color segmentation and edge orientation to detect lanes of roads of any color (especially yellow and white which are the common colors for the lane).

\section{COLOR SEGMENTATION}

In color segmentation [11-12], we will find out the objects or part of the image that were of the lane color. That is the image at the end possesses only those parts of the image which has the lane color (yellow or white). The color segmentation deals with the color feature of lane markings. The method works out with the hue saturation value color model rather than the red green blue color model.

\section{A. Pre-processing}

The pre-processing includes reading the input image, conversion to HSV format and split into individual $\mathrm{H}, \mathrm{S}$ and $\mathrm{V}$ bands. The first step in the process is reading the input image. If the image taken is a gray scale image then the image is read as a two dimensional array. If the input image file contains a true color (RGB) image, it is a three-dimensional array mxnx3. The RGB image is converted into HSV format [13] as hue, saturation and value are properties of a particular color in an image whereas Red, Green and Blue are the primary colors which when combined gives rise to a particular color. Then we segment the areas of a particular color of HSV image by applying thresholds to Hue, Saturation and Value from the HSV image.

\section{B. Threshold and yellow/ white mask}

The values of the Hue, saturation and value of the Lane color are set as the thresholding values. Table 1 describes the set of threshold values are used for yellow and while colored lanes. The values are taken after through experimentation on different types of road images under various conditions.

\section{Boundary removal and labeling the regions by connected components}

We employ the logical and operation of the masks in the above step to obtain the Single Lane colored mask and then apply the colored masks to RGB bands by typecast the yellow object mask/white object mask, as the case may be, into the class of RGB image by using cast function. Now this RGB image obtained is split into individual Red, Green and Blue bands .To each Individual band we multiply the "yellow Objects Mask/ White Objects Mask" so as to obtain masked red green blue images separately. Now we concatenate these masked images into a single masked RGB image. The final masked RGB image consists of the desired lane colored portions only.

TABLE I. THRESHOLD VALUES FOR DETECTING YELLOW AND WHITE COLOR LANES

\begin{tabular}{|c|c|c|c|c|}
\hline \multirow{2}{*}{$\begin{array}{c}\text { Table } \\
\text { Head }\end{array}$} & \multicolumn{2}{|c|}{ Yellow color lane } & \multicolumn{2}{c|}{ White color lane } \\
\cline { 2 - 5 } & Low & High & Low & High \\
\hline Hue & 0.10 & 0.14 & 0.0 & 0.14 \\
\hline
\end{tabular}




\begin{tabular}{|c|c|c|c|c|}
\hline \multirow{2}{*}{$\begin{array}{c}\text { Table } \\
\text { Head }\end{array}$} & \multicolumn{2}{|c|}{ Yellow color lane } & \multicolumn{2}{c|}{ White color lane } \\
\cline { 2 - 5 } & Low & High & Low & High \\
\hline $\begin{array}{c}\text { Saturati } \\
\text { on }\end{array}$ & 0.4 & 1.0 & 0.0 & 0.2 \\
\hline Value & 0.8 & 1.0 & 0.8 & 1.0 \\
\hline
\end{tabular}

V. EDGE ORIENTATION

The lane colored objects that are identified in the color segmentation step are subjected to the edge orientation by using the eccentricity [14] property of shape. After the image is subjected to these steps we finally will be able to detect the lane of any color on the road.

\section{A. Threshold and noise removal}

The masked image obtained at the end of Color Segmentation process is considered as the input image in this step. In the thresholding step [15-16], the input image is converted into a gray scale image. The intensity of a gray scale image varies from 0 to $255(0$ for black and 255 for white). Since the lanes to be detected are in white color, the pixel intensity at the region of the lane is closer to $255(>200)$. Therefore we set a threshold value above 200. The pixels whose intensity is above threshold are made white and the pixels below the threshold are eliminated. Thus a gray scale image is converted into a binary image that has intensity values 0 or 1 of which our lanes are an integral part.

The next step in our process is removal of unnecessary pixels. These unnecessary pixels include the noise and the boundary objects existing in the image. We performed these operations by performing certain morphological operations on the image. We first morphologically open the binary image and eliminate all the connected components of the binary image that have the number of pixels less than the amount specified by us.

\section{B. Remove boundary objects \& labeling the regions}

The next step in the process is identifying the boundaries of the lighter regions inside the binary image. Through this the number of regions existing in the image is identified and is also labeled accordingly. The regions in the image are visualized by assigning the colors to all the regions existing uniquely.

\section{Feature extraction and detecting the lanes}

After identifying different regions inside the binary image, we need to measure the properties like region like Area, Eccentricity, Major Axis Length, Minor Axis Length, Orientation and Perimeter etc., of each and every region of the image in order to find the lanes present in the road image. In this paper we are mainly concerned with the property Eccentricity. The value of eccentricity varies from 0 to 1 . The value 0 indicates that the region is in the form of a circle and if it is 1 , then the region is a straight line. Since our lanes are straight, they may have the eccentricity value closer to 1 . We store the eccentricity values of each region in an array and compare them with a value 0.98 and the regions having eccentricity more than 0.98 are identified and stored separately in another array.

The final step of the system is detecting the lanes. This can be easily done by mapping the original image with the binary image on which the lanes have been identified. We iterate the array of straight lines produced in the above step and mark each pixel of the region being iterated is marked with the required color thus identifying the lanes in the image.

\section{PERFORMANCE ANALYSIS FOR YELLOW COLORED IMAGES AND WHITE COLORED IMAGES}

The images shown in Figure 4 and Figure 5 represent the way to detect the yellow colored lanes and white colored lanes respectively by using the HSV values shown in Table 1 . In Figure 4, we consider yellow colored lane image as input and after applying the said series of steps we have an output image with identified yellow colored lines and in Figure 5, a typical Indian road with white colored lane is considered for the test and after applying the said series of steps, the exact location of the white lines are detected and identified.

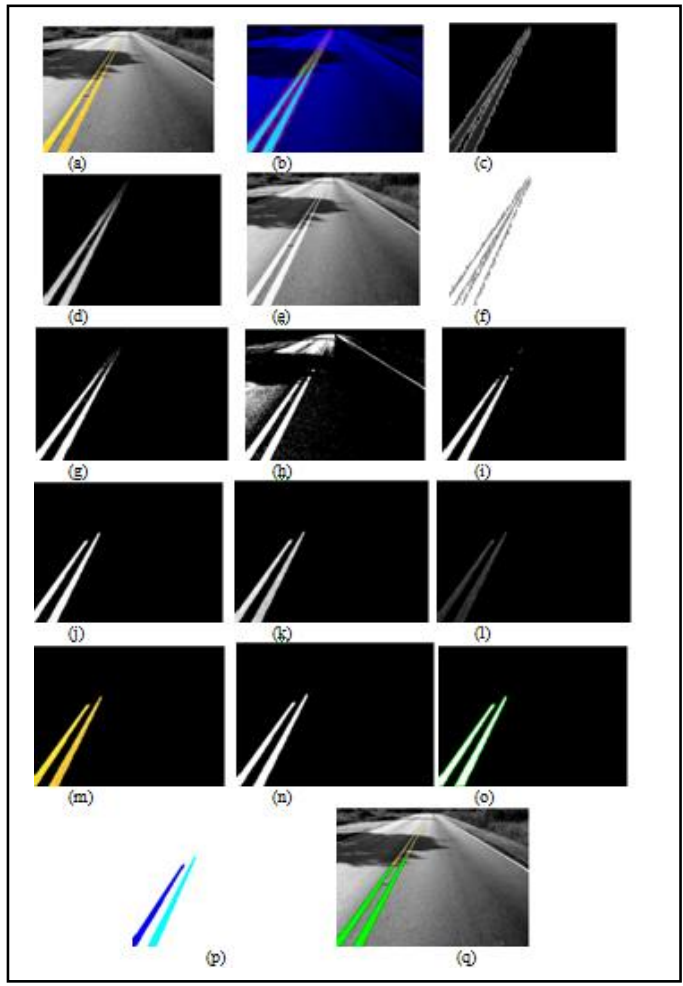

Figure 4. (a) Original Image (b) HSV image(c) Hue Image(d) Saturation image(e) Value image (f) Masked Hue(g) Masked Saturation(h) Masked Value(i) Yellow Masked image (j) Masked Red Image(k) Masked green image(l) Masked blue image(m) Masked yellow image (n) Threshold Image (o) Boundaries identified (p) Regions labeled (q) Identified lanes. 


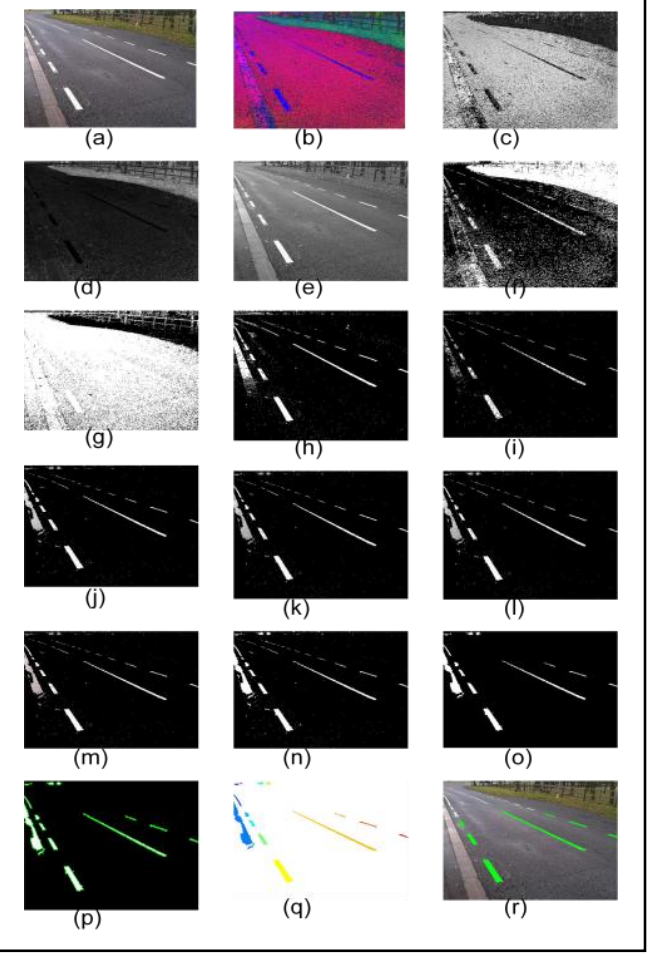

Figure 5. (a) Original RGB Image (b) HSV image (c) Hue Image (d) Saturation Image (e)Value Image (f) Hue Mask (g) Saturation Mask (h) Value Mask (i) White portions left (j) Masked Red Image (k) Masked Green Image (1) Masked Blue Image (m) Masked RGB Image (n) Thresholded Image (o) Noise Removed image (p) Boundaries Identified (q) Regions Labeled (r) Lanes Identified

\section{CONCLUSION}

In this paper, we have developed and implemented a novel algorithm to detect white and yellow colored lanes on the road. The lane detection method is robust and effective in finding the exact lanes by using both color and edge orientations. The main contributions in this paper are the color segmentation procedure identifies the yellow of white colored lanes followed by edge orientation in which the boundaries are eliminated, regions are labeled and finally the lanes are detected. As the height of the camera is relatively constant with respect to the road surface, the road portion of the image can be exclusively cropped by providing the coordinates, so that identifying the lanes becomes much more efficient. The experimental results show the effectiveness of the proposed method in cases of yellow and white colored lanes. The entire work is done in a static way that is on an image. We can extend this to detect lanes in a video.

\section{ACKNOWLEDGMENT}

This work was supported by Major Research Project on Robust non-intrusive multi-modal real-time "vehicle black box" to monitor driver and vehicle state funded by UGC, New Delhi.

\section{REFERENCES}

[1] S Sathees, Man Made Disasters-Road Accidents, Highways Research Station, Chennai

[2] Dinesh Mohan, Omer Tsimhoni, Micheal Sivak, Michael J Flannagan: The University of Michigan Strategic Worldwide Transportation 2020-
ROAD SAFETY IN INDIA: CHALLENGES AND OPPORTUNITIES (2009)

[3] NCRB [National Crime Records Bureau], (2007), Accidental deaths and suicides in India - 2006, New Delhi: Ministry of Home Affairs, National Crime Records Bureau.

[4] Amol Borkar, Monson Hayes and Mark T. Smith: A Template Matching and Ellipse Modeling Approach to Detecting Lane Markers, Lecture Notes in Computer Science (2010)

[5] K. Kluge, S. Lakshmanan: A deformable-template approach to lane detection, in Proc. IEEE Intell. Vehicle Symp (1995)

[6] Y. U. Yim , S. Y. Oh: Three-feature based automatic lane detection algorithm (TFALDA) for autonomous driving, IEEE Trans. Intell. Transp. Syst., vol. 4, no. 4 (2003)

[7] ZuWhan Kim: Lane Detection and Tracking in Challenging Scenarios IEEE TRANSACTIONS ON INTELLIGENT TRANSPORTATION SYSTEMS, VOL. 9, NO. 1 (2008)

[8] M. Bertozzi, A. Broggi: GOLD: a parallel real-time stereo vision system for generic obstacle and lane detection. IEEE Transactions on Image Processing (1997)

[9] F. Diebolt: Reconnaissance des marquages routiers. PhD thesis, L'Univ. Louis Pasteur de Strasbourg, Strasbourg, France(1996)

[10] Yu-Chi Leng , Chieh-Li Chen: Vision-Based Lane Departure Detection System in Urban Traffic Scenes 2010 11th Int. Conf. Control, Automation, Robotics and Vision (2010)

[11] Y. He, H. Wang, B. Zhang, Color-Based Road Detection in Urban Traffic Scenes IEEE Transactions on ITS, vol. 5, pp. 309-318 (2004)

[12] H.Y. Cheng et al : Lane detection with moving vehicle in the traffic scenes IEEE Transactions on ITS, Vol. 7, pp. 571-582(2006)

[13] Jin-Wook Lee, Jae-Soo Cho, Effective lane detection and tracking method using statistical modeling of color and lane edge-orientation in Advanced in Information Sciences and Service Sciences Volume 2, Number 3 (2010)

[14] Amol Borkar, Monson Hayes and Mark T. Smith, A Template Matching and Ellipse Modeling Approach to Detecting Lane Markers Lecture Notes in Computer Science, 2010, Volume 6475/2010, 179-190, DOI: 10.1007/978-3-642-17691-3_17

[15] S. Nedevschi, R. Schmidt, T. Graf, R. Danescu, D. Frentiu, T. Marita, F. Oniga, and C. Pocol : 3D lane detection system based on stereovision in Proc. IEEE Intelligent Transportation Systems Conf., Washington, DC, pp. 161-166 (2004)

[16] D.J. Kang , M.-H. Jung: Road lane segmentation using dynamic programming for active safety vehicles - Pattern Recognition Letters, Vol .24 , pp. 3177-3185 (2003)

[17] Hong Wang Qiang Chen "Real-time lane detection in various conditions and night cases", IEEE Conference on ITS, ITSC '06. pp.1226-1231

[18] Lue-Wei Tsai, Jun-Wei Hsieh, Chi-Hung Chuang, and Kuo-Chin Fan. "Lane Detection Using Directional Random Walks." IEEE Intelligent Vehicles Symposium, 2008.

[19] J. W. Lee, "A machine vision system for lane-departure detection," Com-put. Vis. Image Underst., vol. 86, no. 1, pp. 52-78, Apr. 2002.

[20] M. Aly, "Real time detection of lane markings in urban streets," presented at the IEEE Intelligent Vehicles Symposium, Eindhoven, The Netherlands, 2008.

[21] H. Zhang, et al., "A Novel Lane Detection Algorithm Based on Support Vector Machine," presented at the Progress in Electromagnetics Research Symposium, Hangzhou, China, 2005

[22] Yue Wang,Eam Khwang Teoh, and Dinggang Shen Lane detection and tracking using B-Snake ELSEVIER Image and Vision Computing 22 (2004) 269-280

[23] Road Lane Detection with Elimination of High-Curvature Edges Krzysztof Ślot, Michał Strzelecki, Agnieszka Krawczyńska, Maciej Polańczyk ICCVG 2008 Proceedings of the International Conference on Computer Vision and Graphics

[24] An Effective and Fast Lane Detection Algorithm Chung-Yen Su, GenHau Fan ISVC '08: Proceedings of the 4th International Symposium on Advances in Visual Computing, Part II. 
[25] Chiu, K.-yu, \& Lin, S.-fuu. (2010). A Face Replacement System Based on Face Pose Estimation. International Journal of Advanced Computer Science and Applications - IJACSA, 1(6), 147-156.

[26] Mahjoub, M. A. (2011). Image segmentation by adaptive distance based on EM algorithm. International Journal of Advanced Computer Science and Applications - IJACSA, (Special Issue), 19-25.

[27] Kekre, H. B. (2010). Texture Based Segmentation using Statistical Properties for Mammographic Images. International Journal of Advanced Computer Science and Applications - IJACSA, 1(5), 102-107.

\section{AUTHORS PROFILE}

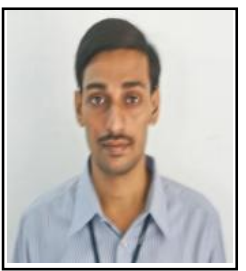

L N P Boggavarapu received Master degree in Computer Science \& Engineering from Acharya Nagarjuna University. Presently he is working as a Lecturer in the Department of Information Technology VR Siddhartha Engineering College, Vijayawada, Andhra Pradesh, India

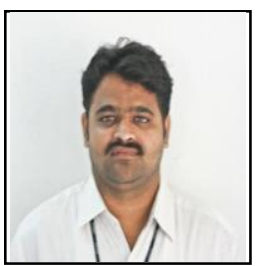

R.S Vaddi received both Bachelor and Master degrees from Acharya Nagarjuna University. Presently he is working as a Lecturer in the Department of Information Technology VR Siddhartha Engineering College, Vijayawada, Andhra Pradesh, India.

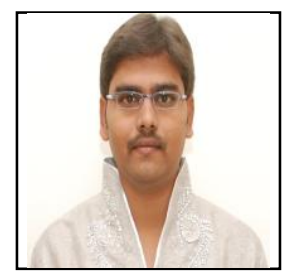

J K Munagala received both Bachelors and Masters from Acharya Nagarjuna University. Presently he is working as Assistant Professor in the Department of Electronics \& Computers, P V P Siddhartha Institute of Technology, Vijayawada, Andhra Pradesh, India.

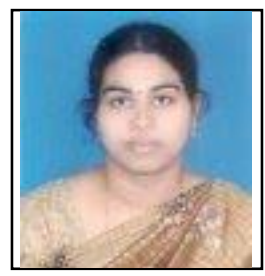

H.D Vankayalapati received Masters Degree from University of Klagenfurt, AUSTRIA. Presently she is working as a Lecture in the Department of Computer Science and Engineering, VR Siddhartha Engineering College, Vijayawada, Andhra Pradesh, India.

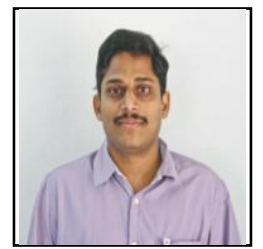

K.R Anne received his Bachelors degree from Bharatiyar University, Masters from University of Hannover, Germany and $\mathrm{PhD}$ from University of Klagenfurt, AUSTRIA. Presently he is working as a Professor in the Department of Information Technology, V R Siddhartha Engineering College, Vijayawada, Andhra Pradesh, India. 